\title{
Retos de la Formación Doctoral: Hacia la Disciplinarización de los Estudios Organizacionales
}

\section{Doctoral Training Challenges: Toward Disciplining Orga- nizational Studies}

Mónica López-Santamaría ${ }^{a}$

\author{
INFORMACIÓN DEL \\ ARTÍCULO \\ Fecha de recepción: 16 de febrero del \\ 2017 \\ Fecha de aceptación: 15 de junio del \\ 2017
}

\footnotetext{
$a$ Doctoranda en el programa de Ciencias de la Dirección, Escuela de Administración de la Universidad del Rosario, Magíster en Derechos Humanos, Universidad Externado de Colombia, Especialista Cultura de Paz y DIH Pontificia Universidad Javeriana Cali, Psicóloga, Universidad del Valle. Docente Escuela de Administración de la Universidad del Rosario.

E-mail: monica.lopezs@urosario.edu.co

* Agradezco al profesor Mauricio Sanabria porque en el marco de la asignatura Epistemología y Metodología de la Ciencia, orientada por él en el Doctorado de Ciencias de la Dirección de la Universidad del Rosario, motivó las reflexiones que hicieron posible este trabajo.
}

\begin{abstract}
Resumen
Teniendo en cuenta el proceso de disciplinarización por el que atraviesa el campo de los estudios organizacionales, este trabajo de revisión teórica trata acerca de los principales retos que enfrenta la formación doctoral en administración: la formación del espíritu científico, la formación en epistemología y la formación en la construcción de teoría. Con el objetivo de fortalecer los hallazgos se analizaron los planes de estudio de los programas doctorales en administración de Colombia y se revisaron las tesis doctorales disponibles en los repositorios de este país, con el fin de rastrear si se hace explícita la declaración del posicionamiento epistemológico en el que se inscriben las investigaciones.
\end{abstract}

\section{Palabras Clave:}

Estudios organizacionales, formación doctoral, espiritu científico, posicionamiento epistemológico, construcción de teoría.

\section{Clasificación JEL: M1}

\begin{abstract}
This paper concentrates on the disciplining process faced by the field of organizational studies. It attempts a theoretical revision of the main challenges faced by doctoral training in management; such challenges are the formation of a scientific spirit, the formation of epistemology, and the formation of a theory construction ability. In order to strengthen findings, doctoral study plans and available doctoral theses in repositories in Colombia were analyzed; the purpose was to ascertain whether declarations of epistemological positioning which research is inscribed on, were actually followed.
\end{abstract}

\section{Keywords}

Organizational studies, doctoral training, scientific spirit, epistemological positioning, theory construction.

JEL Classification: M1 


\section{Introducción}

Según un estudio realizado por Acosta, de la Universidad Nacional de Colombia y Celis, de la Universidad de Estocolmo (Suecia), es posible precisar, entre otros hallazgos relacionados con la formación doctoral en Colombia, que los programas académicos orientados a formar en este nivel han aumentado a partir de 1990. Esto si se tiene en cuenta que para entonces solo se registraban seis programas de esta naturaleza. En 2002 la cifra era de treinta y cuatro, en 2013 se contaba con 197 y para abril de 2015 con 226. Proporcionalmente, se reporta un aumento en el número de estudiantes inscritos a este nivel de formación, que pasó de 2920 en el año 2011 a 3467 en 2013. No obstante, los registros de culminación de estudios reflejan que en el período comprendido entre 1990 y 2011 solo han obtenido el título de doctores 1250 estudiantes (Revista Semana, 2015).

De la misma manera, en el marco de la versión CXXXIII del Consejo Nacional de Rectores celebrado en marzo de 2014 por la Asociación Colombiana de Universidades (ASCUN), se llevó a cabo la revisión de la experiencia de los doctorados en Colombia y se determinó que, a pesar de que en los últimos cinco años se ha presentado un incremento de programas de formación en este nivel, Colombia sigue presentando una cifra baja al respecto. Esto en comparación con países como Brasil, el líder de la región en esta materia. En 2011 esta última nación graduaba 12.217 doctores al año, mientras que la nuestra solo titulaba a 245 .
Ahora bien, al reparar sobre el número de matrículas anuales a programas de doctorado, según campo de conocimiento, se encuentra en las áreas de Economía, Administración, Contaduría y afines una cifra de setenta y ocho estudiantes, obteniendo así un séptimo lugar entre un grupo de ocho áreas de conocimiento. Así mismo, cuando se revisa el número de graduados en este ámbito de formación, se registra una cifra de veinticuatro estudiantes entre 2000 y 2011 (Chaparro, 2014).

En contraste con el estado del proceso de formación doctoral en Administración, se encuentra también que el campo de los Estudios Organizacionales -EO- (Sanabria, Saavedra \& Smida, 2014b) demuestra estar transitando hacia un proceso de disciplinarización (Sanabria, 2006; Sanabria, Saavedra \& Smida, 2014a; Sanabria et al., 2014b) en el que el fortalecimiento de competencias en investigación resulta fundamental.

El panorama que proporcionan las cifras sobre la formación doctoral en Colombia y las consideraciones acerca del desarrollo del campo de los EO plantean cómo, en términos del primer aspecto se configuran varios retos. Estos han venido siendo señalados por varios autores no sólo en el nivel doctoral, sino también en el de pregrado y maestría, así como en la actividad de investigación dentro del campo (Carvajal, 2010; Garcés-Prettel \& Santoya-Montes, 2013; Hernández, Saavedra \& Sanabria, 2006; Romero, 2010; Sanabria, 2007; Sanabria et al., 2014a, 2014b). 
Este trabajo se estructura a partir de la pregunta ¿en qué consisten los principales retos que enfrenta la formación doctoral en administración, en el marco del proceso de disciplinarización de los EO? Se pretende discutir tres retos definidos a partir de la revisión de la literatura. Estos se concretan, a su vez, en tres ámbitos vinculados con la formación, a saber: 1) el espíritu científico, 2) la epistemología y 3) la construcción de teoría. Para cumplir con el objetivo propuesto se comentará primero el proceso de disciplinarización propio del campo de los EO; luego se discutirá sobre los tres retos mencionados antes, trayendo a discusión los datos extraídos de los planes de estudio que orientan los programas doctorales en Colombia y el rastreo de las tesis doctorales disponibles en los repositorios de este país. Finalmente, se dará cuenta de las conclusiones que resultan del análisis de cada uno de los retos tratados.

\section{Disciplinarización del Campo}

La Segunda Revolución Industrial se considera el escenario que favoreció el inicio del proceso de teorización en torno al objeto de estudio de la Administración, ya que es a partir de este momento histórico que la categoría "organización" deja de ser representada a través de su dueño y experimenta un proceso de abstracción que le otorga autonomía y personalidad (Sanabria et al., 2014b). Concretamente, según Augier y March (2011) citados por (Sanabria et al., 2014a), la construcción del campo se abrió paso entre 1945 y 1970 en Estados Unidos. Contexto en el que se experimentó la formalización de los temas de interés de la administración, a partir de la adscripción al método científico con una primacía del enfoque positivista en el ejercicio de la investigación (Sanabria et al., 2014a). Hacia 1970 tendría lugar un proceso similar en el continente europeo, el cual tardó en materializarse en comparación con lo vivido en Norteamérica. Esto debido a los costos de la rehabilitación posbélica derivados de la Segunda Guerra Mundial. Esta corriente de los EO surge en oposición a la propuesta estadounidense, dando cabida a otras formas de aproximación al objeto de estudio (Sanabria et al., 2014a, 2014b). Latinoamérica y particularmente Colombia, experimentan el proceso de configuración del campo cuarenta años después en comparación con los Estados Unidos y veinte con relación a Europa (Sanabria et al., 2014a). Esto permite considerar que en la actualidad, la formación en administración en esta región del mundo se encuentra inevitablemente permeada por estas dos perspectivas de los EO (Sanabria, 2007).

Es posible entonces, a partir de esta revisión del desarrollo del campo, hacer tres aseveraciones: 1) efectivamente este atraviesa por un proceso de disciplinarización (Sanabria, 2006; Sanabria et al., 2014a, 2014b), 2) particularmente en Colombia su desarrollo no alcanza aún un grado de madurez, si se tiene en cuenta el inicio posterior del proceso en comparación con Estados Unidos y Europa (Sanabria et al., 2014a) y 3) la evolución del campo en el último siglo ha sido importante, pese a que aún esta no sea comparable con el desarrollo de otras disciplinas que registran una dinámica distinta (Hernández et al., 2006). 
Ahora bien, el proceso de disciplinarización postula como fundamental el papel de la investigación y, por ende, el rol de los encargados de hacerla posible. Estos deberán esmerarse por alcanzar una aproximación al objeto de estudio muy propia, procurando una producción de conocimiento acorde al contexto y al proceso de desarrollo de cada uno. Así como considerar los asuntos de orden metodológico, ontológico y epistemológico, que fundamentan los cimientos del campo (Sanabria, 2006; Sanabria et al., 2014a, 2014b).

\section{Retos de la Formación doctoral en Administración}

El primer artículo del Decreto 2791 de 1994, que regula entre otros aspectos los requisitos y procedimientos para la creación de programas de doctorado en Colombia, señala que el objetivo de estos consiste en la cualificación de investigadores con capacidad para liderar de forma autónoma este ejercicio, que cuenten con el reconocimiento de la comunidad científica y que aporten al progreso de la ciencia. Así se considera que, al fortalecer la formación del espíritu científico, en epistemología y en lo relativo a la construcción de teoría en los programas doctorales en administración se estará aportando, como corresponde, a la participación activa en la consolidación del proceso de disciplinarización del campo.

\section{La formación del espíritu científico.}

La formación del espíritu científico, tal y como lo plantea Bachelard (2000), no permite que los investigadores mani- manifiesten opiniones acerca de fenómenos que no están al alcance de su comprensión. Quienes lo poseen, defienden la idea de que el conocimiento se construye y que la única forma de abrirle camino es a partir de la formulación de una pregunta. Así las cosas, "el hombre animado por el espíritu científico, sin duda desea saber, pero es por lo pronto para interrogar mejor" (Bacherlard, 2000, p. 19). Por su parte, Alvesson y Sandberg (2013a) indican que las preguntas son el origen fundamental de todas las formas posibles en el proceso de producción de conocimiento. Así la posibilidad de preguntarse acerca de la realidad, la duda sobre el conocimiento plenamente aceptado y la curiosidad, se proponen como un elemento clave en la configuración de ese espíritu científico (Feynman, 2005, 2011).

Sin embargo, Sanabria (2007) advierte que la naturaleza práctica otorgada a la administración, favorece que la capacidad crítica, analítica, teórica y reflexiva se vean marginadas en el proceso de formación desde el nivel de pregrado. Esto dificulta que la imaginación, la capacidad de discusión y la autonomía en la investigación sean plenamente desarrolladas, lo que entorpece la formación del espíritu científico del Administrador de Empresas y, por ende, el proceso de disciplinarización del campo.

\section{La formación en epistemología.}

La formación en epistemología, específicamente en aquella propia del campo, resulta un asunto primordial. Esto si se tiene en cuenta, que el desarrollo de este, ha dado cabida a varios planteamientos $\mathrm{y}$ 
formas de aproximación en relación con su objeto de estudio (Sanabria et al 2014b). Lo anterior, supone la coexistencia de diversas maneras de abordar y concebir a la organización, que han configurado un escenario de "guerra de paradigmas" (Gage, 1989; Jackson \& Carter, 1991). En este sentido, el investigador inscrito al campo de los EO necesita reconocer y comprender la complejidad propia de su objeto de estudio (Cruz, 1982; Hassard, Kelemen \& Cox, 2008; Hernández, Saavedra \& Sanabria, 2007; Kliksberg, 1975; Sanabria, 2006), para darle valor a la discusión epistemológica iniciada por Burrell y Morgan (1979), cuya concepción de paradigma difiere de la planteada por Kuhn (2004). Así en el campo de los EO el paradigma representa diferentes escuelas de pensamiento, que se acercan a la realidad de formas distintas con una visión compartida del mundo representada en el mismo objeto de estudio (Morgan, 1980).

La postura paradigmática, tal y como lo argumentan Parker y McHugh (1991), no puede reducirse a ser empleada como un marco de referencia empírico, ya que los paradigmas representan un fundamento de orden ontológico, epistemológico y también metodológico profundo, que configura un sistema de creencias al que se adhiere una comunidad científica en particular. Siguiendo a Bachelard (2000) "el epistemólogo toma los hechos como ideas, insertándolas en un sistema de pensamiento" (p. 20). Esto trae como consecuencia que las diferentes visiones que tienen los investigadores acerca del mundo le dan forma a diversos intereses de conocimiento. Cada una de estas de- fiende además unos supuestos sobre la realidad social y las formas de comprenderla (Morgan \& Smircich, 1980). Así en la labor investigativa se interactúa y se construye la realidad a partir de una visión paradigmática (Sanabria, 2006).

Además de ser consciente de la importancia de definir y hacer explícita la postura epistemológica, es necesario que el estudiante de doctorado esté seguro que tiene clara la diferencia entre el nivel epistemológico y el nivel metodológico. Esta se puede comprender cuando Mir y Watson (2000), afirman que los métodos cuantitativos podrían ser desplegados en una investigación de orden constructivista y a su vez los métodos cualitativos podrían ser desplegados en el marco de una investigación de orden realista. Lo que muestra que no necesariamente todos los estudios cuantitativos están inscritos en una epistemología positivista.

Ahora bien, en virtud de explorar la formación en epistemología que promueven los programas doctorales en administración en Colombia, se llevó a cabo una revisión de los planes de estudio de los nueve programas vigentes, a saber: Universidad Eafit, Universidad de los Andes, Universidad del Valle, Universidad del Rosario, Universidad EAN, Universidad del Norte, Universidad de Medellín, Universidad Externado de Colombia y Universidad Simón Bolívar. Se encontró que seis de estos hacen explícita una asignatura con el nombre de epistemología (Universidad Eafit, Universidad del Valle, Universidad del Rosario, Universidad EAN, Universidad del Norte y Universidad Simón Bolívar). A pesar 
de que se reconoce que este componente de formación puede estar contemplado en las asignaturas denominadas bajo la categoría de "investigación", el ejercicio de exploración arroja que las dos terceras partes de los programas doctorales han destinado créditos académicos a la formación en esta materia, lo que se traduce en un panorama alentador. Sin embargo, al explorar las tesis doctorales disponibles a través de los repositorios se encontró que, de catorce tesis consultadas, cuatro hacen explícita la postura epistemológica del investigador considerando este aspecto como un apartado dentro del capítulo de diseño metodológico. Ahora bien, de las cuatro tesis, sólo una menciona los supuestos del paradigma en el que se inscribe la investigación y determina que desde allí se edifica su estudio. Las demás incurren en el error de traslapar la postura epistemológica con el enfoque metodológico, lo que sugiere que este aspecto aún requiere ser fortalecido en la formación doctoral.

Según Márquez (2008) citado por Garcés-Prettel y Santoya-Montes (2013) la tesis doctoral debe considerar indispensablemente cuatro componentes (entre los cuales la postura epistemológica es considerada). Estos son: 1) aproximación al objeto de estudio, que se define a partir de los objetivos y la pregunta de investigación; 2) el soporte epistemológico, que se construye a partir de los supuestos de cada paradigma; 3) el contexto conceptual, que demanda una revisión crítica de la literatura relacionada con el fenómeno a estudiar y 4) el soporte metodológico, que incluye el diseño, método, procedimiento y técnicas a emplear en el ejercicio de investigación. Este último debe ser coherente con el soporte epistemológico declarado. Así las cosas, la epistemología actúa como el mecanismo para señalar los límites y los supuestos de los que parte la concepción de la realidad y del conocimiento a los que se adhiere la comunidad científica que los emite (Johnson \& Duberley, 2000).

\section{La formación en construcción de teoría}

La construcción de teoría constituye el tercer pilar en el proceso de formación de los estudiantes de doctorado. En particular cuando se llama la atención sobre el hecho de que aquellos inscritos al campo de los EO, alcanzan el nivel de comprensión de los modelos teóricos existentes, a veces con dificultad, y se dedican a replicarlos. Lo anterior, sin incursionar necesariamente en su propia comprensión del fenómeno organizacional, con el fin de edificar sus propios modelos (Sanabria, 2006). Al respecto, Byron y Thatcher (2016) llevaron a cabo una investigación para explorar la formación en construcción de teoría en el nivel doctoral, partiendo de las siguientes preguntas: 1) ¿qué es y qué no es una teoría?, 2) ¿Hay qué preocuparse por la teoría?, 3) ¿de dónde vienen las nuevas teorías?, 4) ¿cómo se construye una buena teoría? y 5) ¿cómo se obtiene una teoría publicada? A partir de las cuales se llevará a cabo la respectiva discusión sobre este tema.

En términos generales, Byron y Thatcher (2016) encontraron que, según lo reportado por los profesionales entrevistados, 
la lectura se presenta como un requisito obligatorio en el proceso de aprendizaje de construcción de teoría, además, esta formación se favorece tanto por medio de estrategias estructuradas, como no estructuradas. Ahora bien, algunos programas de doctorado dedican un espacio de formación para este tema, pero por lo general este entrenamiento ocurre a partir de estrategias no estructuradas como tutorías o talleres de desarrollo.

Respecto a qué es y qué no es una teoría, es posible apreciar en el número 3 , volumen 40, de la revista Administrative Science Quarterly tres artículos dedicados a este tema (DiMaggio, 1995; Sutton \& Staw, 1995; Weick, 1995), a partir de los cuales se puede apreciar un panorama más claro acerca de lo que se concibe y lo que no como teoría. Por otro lado, respecto a este tema, varios autores destacan que es importante reconocer cómo se entiende la acción de aporte teórico y el proceso para llevarlo a cabo (Corley \& Gioia, 2011; Cornelissen \& Durand, 2014). Por su parte, Byron y Thatcher (2016) encontraron que los profesores entrevistados en su estudio, advirtieron sobre la importancia de motivar a los estudiantes para que identifiquen los supuestos a partir de los cuales se construyen las teorías que fundamentan sus investigaciones. Esto con el objetivo de impulsar la formulación de nuevas proposiciones que las enriquezcan o en su defecto las refuten.

Para construir una buena teoría resulta fundamental en primera instancia comprender a qué se refieren con "buena", para lo cual se indicará que una teoría con estas características debe ser: 1) interesante (Weick, 1989), entendiéndola como aquella que incursiona en el proceso de negar ciertos supuestos teóricos que se habían dado por sentado (Davis, 1971) y 2) debe ser catalogada como seductora, porque ha cumplido con ciertas características, más allá de su veracidad y validez (Davis, 1986).

En relación con la construcción de teoría, se considera indispensable que el investigador reconozca también las posibilidades con las que cuenta para maniobrar el proceso de investigación: por un lado, construir preguntas en oposición al procedimiento habitual catalogado como "gap-spotting" (identificación de brechas), a partir de la detección de anomalías teóricas, que permitan la creación y resolución de misterios (Alvesson \& Kärreman, 2007) y por el otro, privilegiando la construcción de teorías, a partir de modelización matemática y la construcción de modelos causales (Donaldson, Qiu \& Luo, 2013).

Finalmente, frente al último punto relacionado con la publicación de teorías, la reflexión se orienta a la pertinencia y contribución del resultado de las investigaciones. Al respecto, por un lado, se encuentran posturas críticas en relación con el nivel de impacto y contribución de las publicaciones (Alvesson \& Sandberg, 2013b, 2014) y por el otro, se observa un panorama más alentador en cuanto al proceso de construcción de teoría en el campo (Colquitt \& Zapata-Phelan, 2007). Sanabria et al (2014a) señalan, en torno al contexto colombiano que: 
El análisis de las publicaciones de los investigadores en administración en el país y del concepto de los expertos en la materia permite constatar que están dadas las condiciones para lograr un mayor crecimiento de la producción científica nacional en el campo, y para mejorar la calidad de los resultados de las investigaciones (p. 19).

\section{Conclusiones}

Retomando, es posible precisar que el campo de los EO atraviesa por un proceso de disciplinarización, que en Colombia se torna mucho más incipiente a causa de su posterior incubación en comparación con lo acontecido en E.U y Europa. De esta manera, el papel de la investigación se posiciona como fundamental para avanzar en este proceso. En consecuencia, el rol de los estudiantes de doctorado como futuros investigadores enfrenta retos, a los que debe responder para contribuir de manera eficiente en la construcción del campo.

Como primera medida la formación del espíritu científico implica promover la capacidad de formulación de preguntas investigables entre la población de estudiantes de doctorado y transmitir la importancia de la pregunta como punto de partida en el proceso de producción de conocimiento. Así la virtud de poner en duda lo establecido, la curiosidad, la intuición y la heurística se proponen como herramientas fundamentales para el investigador.

La formación en epistemología debe- rá garantizar que el estudiante de doctorado reconozca y comprenda la complejidad del objeto de estudio de la administración, que diferencie la concepción de paradigma propia de la ciencia normal a aquella adscrita a las ciencias sociales y que cuente con la competencia para definir y hacer explícito el posicionamiento epistemológico que orientará su labor investigativa. Finalmente, resulta fundamental en este nivel de formación que el investigador tenga clara la diferencia entre los aspectos epistemológico y los metodológicos.

La formación en construcción de teoría le debe permitir al estudiante de doctorado saber qué es y qué no es una teoría, tener claro que para construir una buena teoría se requiere que esta sea interesante, seductora y responda a un proceso de reflexividad. Así mismo, resulta indispensable que en este nivel de formación el estudiante identifique que es posible construir teoría por vía de identificación de brechas en el conocimiento, o por medio de la identificación de anomalías en las teorías en las que pretende basar su estudio.

De esta manera, los retos señalados en torno a la formación doctoral en Administración reflejan la importancia de los procesos de actualización curricular y autoevaluación constante, que favorezcan los ajustes necesarios para continuar contribuyendo a la disciplinarización del campo de estudio.

\section{Referencias}

Alvesson, M., \& Kärreman, D. (2007). 
Constructing mystery: Empirical matters in theory development. Academy of Management Review, 32(4), 1265-1281.

Alvesson, M., \& Sandberg, J. (2013a).

Constructing research questions: Doing interesting research. Londres: SAGE.

Alvesson, M., \& Sandberg, J. (2013b).

Has management studies lost its way? Ideas for more imaginative and innovative research. Journal of Management Studies, 50(1), 128-152.

Alvesson, M., \& Sandberg, J. (2014).

Habitat and habitus: Boxed-in versus box-breaking research. Organization Studies, 35(7), 967-987.

Bachelard, G. (2000). La formación del espiritu científico. Contribución a un psicoanálisis del conocimiento objetivo. México: Siglo XXI.

Burrell, G., \& Morgan, G. (1979). Sociological paradigms and organisational analysis: Elements of the sociology of corporate life. Londres: Heinemann Educational.

Byron, K., \& Thatcher, S. M. B. (2016). Editors comments: "What I know now that I wish I knew then" teaching theory and theory building. Academy of Management Review, 41(1), 1-8.
Carvajal, J. G. (2010). Algunos problemas de la investigación en administración. En G. Calderón \& G. A. Castaño (Eds.), Investigación en administración: conocimiento para el bienestar de las personas y el desarrollo de las organizaciones (pp. 15-22). Manizales: Universidad Nacional de Colombia.

Colombia. Ministerio de Educación. Decreto Número 2791 por el cual se establecen los requisitos y procedimientos para la creación de programas de Doctorado, se crea la Comisión Nacional de doctorados y se fijan plazos de evaluación de tales programas (22 diciembre 1994).

Colquitt, J. A., \& Zapata-Phelan, C. P. (2007). Trends in theory building and theory testing: A five-decade study of the Academy of Management Journal. Academy of Management Journal, 50(6), 1281-1303.

Corley, K. G., \& Gioia, D. A. (2011). Building theory about theory building: What constitutes a theoretical contribution? Academy of Management Review, 36(1), 12-32.

Cornelissen, J. P., \& Durand, R. (2014). Moving forward: Developing theoretical contributions in management studies. Journal of Management Studies, 51(6), 995-1022. 
Cruz, F. (1982). Hacia una redefinición del concepto de organización. Cuadernos de Administración, 6(1), 7-64.

Chaparro, F. (2014). La experiencia de Colombia en la formación doctoral. CXXXIII Consejo Nacional de Rectores, Asociación Colombiana de Universidades (ASCUN). Cartagena-Colombia.

Davis, M. S. (1971). That's interesting!: Towards a phenomenology of sociology and a sociology of phenomenology. Philosophy of the Social Sciences, 1(2), 309-344.

DiMaggio, P. J. (1995). Comments on "What Theory is Not". Administrative Science Quarterly, 40(3), 391-397.

Donaldson, L., Qiu, J., \& Luo, B. N. (2013). For rigour in organizational management theory research. Journal of Management Studies, 50(1), 153-172.

Feynman, R. P. (2005). The making of a scientist. En C. C. Lundberg \& C. A. Young (Eds.), Foundations for inquiry: Choices and trade-offs in the organizational sciences (pp. 18-24). Stanford: Stanford Business Books.

Feynman, R. P. (2011). What is science? Resonance: Journal of Science Education, 16(9), 860-873.
Gage, N. (1989). The paradigm wars and their aftermath: A "historical" sketch of research and teaching since 1989. Educational Research, 18(1), 4-10.

Garcés-Prettel, M. E., \& Santoya-Montes, Y. E. (2013). La formación doctoral: expectativas $\mathrm{y}$ retos desde el contexto colombiano. Universidad de la Sabana, 16(2), 283-294.

Hassard, J., Kelemen, M., \& Cox, J. W. (2008). Disorganization theory: Explorations in alternative organizational analysis. Londres: Routledge.

Hernández, A. G., Saavedra, J. J., \& Sanabria, M. (2006). La formación administrativa en Colombia: el caso de las maestrías. Revista Facultad de Ciencias Económicas: Investigación y Reflexión, 14(2), 21-38.

Hernández, A. G., Saavedra, J. J., \& Sanabria, M. (2007). Hacia la construcción del objeto de estudio de la administración: una visión desde la complejidad. Revista Facultad de Ciencias Económicas: Investigación y Reflexión, 15(1), 91-112.

Jackson, N., \& Carter, P. (1991). In defence of paradigm incommensurability. Organization Studies, 12(1), 109-127. 
Johnson, P., \& Duberley, J. (2000). Understanding management research: An introduction to epistemology. Londres-Thousand Oaks: SAGE.

Kliksberg, B. (1975). El pensamiento organizativo: del taylorismo a la teoría de la organización. La administración científica en discusión. Buenos Aires: Paidos.

Kuhn, T. S. (2004). La estructura de las revoluciones científicas. México: Fondo de Cultura Económica.

Mir, R., \& Watson, A. (2000). Strategic management and the philosophy of science: The case for a constructivist methodology. Strategic Management Journal, 21(9), 941-953.

Morgan, G. (1980). Paradigms, metaphors, and puzzle solving in organization theory. Administrative Science Quarterly, 25(4), 605-622.

Morgan, G., \& Smircich, L. (1980). The case for qualitative research. Academy of Management Review, 5(4), 491-500.

Parker, M., \& McHugh, G. (1991). Five texts in search of an author: A response to John Hassard's 'Multiple paradigms and organizational analysis'. Organization Studies, 12(3), 451-456.
Revista Semana (20 de febrero de 2015). Colombia se raja en formación doctoral. Recuperado de: http://www.semana.com/educacion/articulo/colombia-formacion-doctoral/418514-3

Romero, L. E. (2010). Investigación en administración en América Latina ¿un futuro promisorio? En G. Calderón \& G. A. Castaño (Eds.), Investigación en administración: conocimiento para el bienestar de las personas y el desarrollo de las organizaciones (pp. 23-28). Manizales: Universidad Nacional de Colombia.

Sanabria, M. (2006). El modelo ecuménico para la comprensión organizacional: una propuesta teórica favorable a la comprensión del sistema cognoscitivo propio de la administración y de su construcción como disciplina. Universidad \& Empresa, 8(11), 182-236.

Sanabria, M. (2007). Conformación actual del campo administrativo en Colombia: una mirada a partir de los programas de formación. Revista Facultad de Ciencias Económicas: Investigación $y$ Reflexión, 15(2), 147-175.

Sanabria, M., Saavedra, J. J., \& Smida, A. (2014a). La construccion de sentido de los investigadores en gestion en Colombia acerca de su campo de conocimiento. Innovar, 24(54), 7-25. 
Sanabria, M., Saavedra, J. J., \& Smida, A. (2014b). Los estudios organizacionales ('organization studies'): fundamentos, evolución y estado actual del campo. Bogotá: Editorial Universidad del Rosario.

Sutton, R. I., \& Staw, B. M. (1995).

What theory is not. Administrative

Science Quarterly, 40(3), 371-384.।

Weick, K. E. (1995). What theory is not, theorizing is. Administrative Science Quarterly, 40(3), 385-390.

Whetten, D. A. (2002). Modelling- astheorizing: A systematic methodology for theory development. En D. Partington (Ed.), Essential Skills for Management Research (pp. 45-71). London: SAGE. 